\title{
Should there be greater exposure to interventional radiology in the undergraduate curriculum?
}

This article was published in the following Dove Press journal:

Advances in Medical Education and Practice

\author{
Utkarsh Ojha' \\ Raihan Mohammed ${ }^{2}$ \\ Sayinthen Vivekanantham ${ }^{3}$ \\ 'Faculty of Medicine, Imperial College \\ London, London, ${ }^{2}$ Department of \\ Medicine, University of Cambridge, \\ Cambridge, ${ }^{3}$ University of Aberdeen, \\ Aberdeen, UK
}

\begin{abstract}
Medical imaging has been one of the most revolutionary innovations in medicine. Today, as health care professionals shift their focus toward more sophisticated technology and minimally invasive procedures, interventional radiology (IR) has become a rapidly expanding specialty. Despite these advances, there is a lack of doctors specializing in this field. A growing body of evidence suggests that the low number of applicants for posts may be due to poor exposure to the specialty at medical school. In this article, we outline the importance of IR in today's health care system. Next, we evaluate the evidence that there is a lack of knowledge of IR not only among medical students in the UK but globally. We further discuss how a more effective incorporation of IR in the undergraduate curriculum can enhance medical students' interest in the field and subsequently increase the number of doctors specializing in IR. Finally, we suggest alternative strategies to gauge medical students' interest in IR, including teaching via e-learning and virtual reality.
\end{abstract}

Keywords: interventional radiology, diagnostic imaging, innovation, medical education, e-learning, virtual reality

\section{Introduction}

On 15 February 2017, the Royal College of Radiologists (RCR) announced that the lack of doctors specializing in interventional radiology (IR) meant only two of the 12 health boards in Scotland offered a 24-hour cover for emergency pinhole procedures, putting patient safety at risk. ${ }^{1}$ According to the British Society of Interventional Radiologists (BSIR), an extra 222 consultants are still needed to meet the target of one interventional radiologist on call for every five on-call doctors in all acute trusts. ${ }^{2}$ IR was officially granted a subspecialty status in 2010 and since then, the number of IR procedures in the UK has increased by over $50 \%{ }^{2}$ Interventional radiologists undertake both vascular and non-vascular interventions. Vascular treatments include dilating narrowed arteries, preventing hemorrhages, and breaking blood clots in the veins. Non-vascular interventions involve shrinking tumors and removing gall stones and kidney stones. ${ }^{3}$ It is widely recognized that IR is a minimally invasive technique which provides many advantages for both patients and health care systems. These benefits include: lower costs than invasive procedures, greater comfort for patients, and shorter hospital stays. ${ }^{3}$ Patients who undergo IR procedures report less pain than those undergoing surgical interventions. Previously, surgical interventions were the only treatment option for many conditions. Consequently, many general practitioners referred their patients to surgeons. However, surgeons are not fully informed about the
Correspondence: Utkarsh Ojha Imperial College School of Medicine, Sir Alexander Fleming Building, Imperial College London, Exhibition Road, SW7 2AZ, London, UK

Tel +447759667685

Email utkarsh.ojhal3@imperial.ac.uk 
variety of minimally invasive procedures on offer. Therefore, it is vital that patients also consult interventional radiologists about these minimally invasive procedures and consider all treatment options. ${ }^{4}$ Consequently, IR has become an increasingly relevant field. Furthermore, IR provides unique research opportunities for evaluating new technologies and therapeutic procedures which enhance patient safety. Despite these advances, medical students' knowledge in this field remains poor. ${ }^{5}$

The reasons for unfilled places remain contentious. However, evidence suggests that the lack of specialization in this field may be due to poor exposure at medical school. Here, we begin by discussing the evidence for poor exposure of IR in the undergraduate curriculum and highlighting our own experience of IR at medical school. Next, we discuss the evidence suggesting that better exposure to the specialty in the undergraduate curriculum may increase the number of doctors applying for IR jobs. Finally, we suggest alternate strategies to increase medical students' interest in IR.

\section{The medical student experience}

The RCR encourages trainees to understand interventional procedures required in patients as part of its trainee curriculum. ${ }^{6}$ Exposure to radiology varies across medical schools. In some institutions, radiology is taught as an independent subject, whilst others integrate radiology with relevant modules including clinical anatomy and physiology. ${ }^{7-9}$ In the latter structure, radiology is often underrepresented in examinations and students therefore have less onus for gaining exposure to the specialty as a whole. ${ }^{10}$ Data from the European Society of Radiology ${ }^{10}$ demonstrate that the average number of teaching hours for IR (5.3 hours) is much less than radiological anatomy (17.5 hours) and radiological techniques (13.7 hours). Atiiga et al ${ }^{11}$ investigated the knowledge and interest of final year medical students in England about IR. One hundred and sixty-seven students out of 220 were able to correctly determine the roles and responsibilities of an interventional radiologist. The majority (55.5\%) felt that their knowledge of IR was less compared to other specialties. Lack of interest and understanding of IR was the most commonly reported reason for students not seeking to pursue a career in IR. Seventy percent of students expressed that they would like more exposure to the specialty at medical school. Evidence shows that knowledge of IR is not only lacking among medical students in the UK, but also globally. O'Malley and Athreya ${ }^{12}$ assessed Canadian medical students' awareness and exposure to IR. The group found that 53 out of 103 respondents claimed they had "poor" knowledge of the specialty and only $18 \%$ claimed they would consider IR as a future career choice. Similarly, Alshumrani ${ }^{13}$ investigated the knowledge of medical students in Saudi Arabia about IR. The study distributed an anonymous questionnaire with 25 questions about the specialty to 119 students, of whom 42 responded. The results showed that the majority of medical students $(52 \%)$ felt that their knowledge of the field was poor, and the most common reason (43\%) for students not considering a career in IR was lack of knowledge.

In our experience as medical students, exposure to radiology itself is relatively short compared to other specialties. Radiological teaching at medical school entails interpreting basic radiographs of the chest, abdomen, and limbs. These are infrequently tested during our practical exams. Moreover, during radiology rotations there is limited teaching allocation to IR. As a result, the relatively short duration of IR attachments at medical school do not allow us to fully appreciate the range of medical conditions that are applicable to IR. Students considering a career in IR must therefore gain exposure outside of the undergraduate curriculum by either shadowing specialists, conducting research in the field, or attending conferences and events in IR. Furthermore, there is an abundance of societies at our medical schools. However, we find that a dedicated radiology and IR society is dormant compared to other more prominent societies such as the surgical society, pathology society, and medical education society. Therefore, the student body is less exposed to learning opportunities in IR compared to other specialties.

\section{Increasing awareness of IR by reforming the undergraduate curriculum}

Some medical students enter medical school having already decided which field they would like to pursue. However, for a vast majority, exposure to specialties and indeed the timing of the exposure can significantly impact their future career choices. ${ }^{14}$

A study by Nissim et a ${ }^{15}$ investigated the extent to which medical students are exposed to IR in medical schools. The authors also assessed students' knowledge of the field and their interest in pursuing IR as a career choice, in a cohort of 729 students. Fifty-eight percent of respondents expressed their interest in a hands-on career. However, just $12.7 \%$ were interested in a career in IR and only 5.5\% of students stated they had participated in an IR rotation. The investigation also reported that students who possessed greater understanding of a career in IR were those who had been exposed to IR rotations. Taken together, the data from this group highlight that 
improved understanding of IR amongst medical students can potentially be enhanced by increasing exposure to the specialty at medical school. However, it must be acknowledged that factors beyond medical education can influence future career choices. ${ }^{16}$ Whilst the study cannot make direct connections between medical education and the projected shortage of IR posts, the authors provide a compelling argument for increasing awareness of IR at medical school.

The need for a dedicated undergraduate IR curriculum is further supported by Shaikh et al, ${ }^{5}$ who designed and distributed a questionnaire to 309 medical students before and after a 10-hour teaching curriculum. The results showed that knowledge of IR clinical roles increased after the teaching session. Interestingly, the number of students who said they would consider a career in IR increased from $60 \%$ to $73 \%$. The results of this study suggest that incorporation of an effective IR curriculum not only raises awareness of, but can also increase enthusiasm for pursuing the specialty as a future career. However, this study is subject to response bias since only those students initially interested in IR would be inclined to complete the questionnaire. Moreover, the anonymous nature of the survey prevents the authors from matching each participant's pre- and post-lecture responses.

In another study, Leong et $\mathrm{al}^{17}$ aimed to assess the knowledge and views of final-year medical students regarding various aspects of IR. The group distributed an electronic survey consisting of 11 questions to 675 students. A total of 234 students replied to the survey, of which $63 \%$ indicated that their knowledge of IR in general was poor. Whilst the majority of respondents felt that the future prospects of IR were "good", less than $50 \%$ were willing to consider IR as a career. The reason for this is speculative. Some suggest that diagnostic radiology itself is an attractive specialty, ${ }^{18}$ therefore trainees are less inclined to pursue IR. Conversely, others argue that the majority of students do not consider radiology as a career choice due to its lack of patient contact. ${ }^{19}$ As a result, they mistakenly also group IR into the same category, thus failing to recognize the immense patient contact the specialty offers.

The aforementioned studies all suggest that appropriate and accurate exposure to IR at medical school is required for students to make fully informed decisions regarding their career choices.

\section{Alternative strategies}

Whilst undergraduate curriculum reforms allow medical students to gain a better insight and appreciation of IR, this strategy has several drawbacks. Shah ${ }^{20}$ argued that teaching time would have to be taken away from the core specialties in order to accommodate teaching for more niche and new specialties. Furthermore, the larger and core specialties require a greater workforce, and the majority of graduates are thus more likely to enter these specialties rather than newer ones like IR.

\section{Raising awareness of IR at medical school can also be achieved by other means}

Appointing student representatives at institutions that inform the student body of upcoming meetings, conferences, and competitions related to IR can also be an effective way of gauging students' interest. Governing bodies like BSIR and RCR could encourage students to set up IR societies at their universities and work in close partnerships with them. These organizations could further support students by providing scholarships, grants, and funding to carry out specialist modules, intercalated BSc or electives within IR. This strategy was recently adopted and proved promising by the Royal College of Psychiatrists to manage the current shortfall of psychiatrists. ${ }^{21}$

The European Society of Radiology guides educators to keep radiological teaching as clinically relevant as possible. ${ }^{22}$ This is further echoed by BSIR's recent publication ${ }^{23}$ which outlines that medical schools should teach anatomy and physiology relevant to interventional procedures, the basic principles of IR techniques, and management of specific clinical cases. Several studies have incorporated imaging techniques during dissection classes to enhance students' knowledge and understanding of diagnostic imaging. Lufler et $\mathrm{al}^{7}$ examined whether the use of cadaveric computed tomography (CT) scans during anatomy lessons was positively associated with performance in the anatomy course. The study demonstrated that students who used CT scans scored significantly higher in practical exams than those who did not. These results were also replicated by Paech et $\mathrm{al}^{8}$ who reported that students using CT scans during anatomy lessons scored $27 \%$ higher in anatomy than those who did not. Several studies ${ }^{9,24}$ have also concluded that as clinical medicine becomes more digital, new methodologies must be employed in order to teach radiology.

Moreover, the use of e-learning to deliver radiology teaching has shown great promise. E-learning involves the use of Internet technology to provide content and knowledge; e-learning allows students to learn in a more stressfree and relaxed environment and enables them to tailor their experience to meet their personal learning outcomes. 
Computer-based radiology teaching is particularly effective and easy to implement due to the visual nature of the specialty. Salajegheh et $\mathrm{al}^{25}$ examined the use of e-learning to increase knowledge in X-ray interpretation in 150 firstand second-year medical students. The authors reported that compared to a control group, e-learning significantly improved the knowledge and skills of X-ray interpretation among medical students.

In addition, medical schools have also piloted incorporating virtual reality to teach anatomy. Codd and Choudhury ${ }^{26}$ described the use of 3D imaging to construct anatomical figures to teach anatomy. The authors assigned medical students to three groups: students with no prior knowledge, students using traditional methods using dissection and textbooks, and a test group, who were taught using e-resources. The authors found that the test group's score in the anatomical exam was significantly higher than the other two groups. This new method of teaching received much positive feedback from both students and faculty members and was shown to be compliant with traditional teaching methods. ${ }^{26}$ It is therefore conceivable that adopting such techniques to teach $\operatorname{IR}^{27}$ can highlight to students the immense innovation in this field and increase their enthusiasm for pursuing the specialty in the future.

\section{Conclusion}

The digitalization of the health care profession has opened new opportunities to educate medical students about this exciting and evolving field. Given the lack of awareness amongst medical students and also the increasing demands for interventional radiologists, greater efforts must be made at the undergraduate stage to promote the specialty.

\section{Author contributions}

UO, RM, and SV contributed toward data analysis, drafting, and revising the paper and agree to be accountable for all aspects of the work.

\section{Disclosure}

The authors report no conflicts of interest in this work.

\section{References}

1. Cramb A. Doctors warn Scottish radiology "on the brink of collapse". The Telegraph. 2017 Feb 15. Available from: http://www.telegraph. co.uk/news/2017/02/15/doctors-warn-scottish-radiology-brink-collapse/. Accessed November 16, 2017.

2. Securing the future workforce supply: Clinical Radiology Stocktake. Centre for workforce intelligence; 2012. Available from: https:// www.gov.uk/government/uploads/system/uploads/attachment_data/ file/507372/CfWI_Clinical_Radiology_Stocktake_2012.pdf. Accessed September 15, 2017
3. British Society of Interventional Radiology [homepage on the Internet]. What is Interventional Radiology? BSIR. Available from: http://www. bsir.org/patients/what-is-interventional-radiology. Accessed November 16, 2017.

4. cirse.org [homepage on the Internet]. Innovation education intervention. CIRSE; 2017. Available from: http://www.cirse.org/index.php?pid=4. Accessed November 16, 2017.

5. Shaikh M, Shaygi B, Asadi H, Thanaratnam P, Pennycooke K, Mirza M, Lee $M$. The introduction of an undergraduate interventional radiology (ir) curriculum: impact on medical student knowledge and interest in IR. Cardiovasc Intervent Radiol. 2015;39(4):514-521.

6. rcr.ac.uk [homepage on the Internet]. Undergraduate curriculum. Royal College of Radiologists. Available from: https://www.rcr.ac.uk/clinicalradiology/undergraduate-curriculum. Accessed November 16, 2017.

7. Lufler RS, Zumwalt AC, Romney CA, Hoagland TM. Incorporating radiology into medical gross anatomy: does the use of cadaver CT scans improve students' academic performance in anatomy? Anat Sci Educ. 2010;3(2):56-63.

8. Paech D, Giesel F, Unterhinninghofen R, Schlemmer H, Kuner T, Doll S. Cadaver-specific CT scans visualized at the dissection table combined with virtual dissection tables improve learning performance in general gross anatomy. Eur Radiol. 2016;27(5):2153-2160.

9. McNiesh L, Madewell JE, Allman RM. Cadaver radiography in the teaching of gross anatomy. Radiology. 1983;148(1):73-74.

10. European Society of Radiology (ESR). Undergraduate education in radiology. A white paper by the European Society of Radiology. Insights Imaging. 2011;2(4):363-374.

11. Atiiga P, Drozd M, Veettil R. Awareness, knowledge, and interest in interventional radiology among final year medical students in England. Clin Radiol. 2017;72(9):795.e7-795.e12.

12. O'Malley L, Athreya S. Awareness and level of knowledge of interventional radiology among medical students at a Canadian institution. Acad Radiol. 2012;19(7):894-901.

13. Alshumrani GA. Awareness of interventional radiology among final-year medical students and medical interns at a university in Southwestern Saudi Arabia. Saudi Med J. 2013;34(8):841-847.

14. Takeda Y, Morio K, Snell L, Otaki J, Takahashi M, Kai I. Characteristic profiles among students and junior doctors with specific career preferences. BMC Med Educ. 2013;13:125.

15. Nissim L, Krupinski E, Hunter T, Taljanovic M. Exposure to, understanding of, and interest in interventional radiology in American medical students. Acad Radiol. 2013;20(4):493-499.

16. Pianosi K, Bethune C, Hurley KF. Medical student career choice: a qualitative study of fourth-year medical students at Memorial University, Newfoundland. CMAJ Open. 2016;4(2):E147-152.

17. Leong S, Keeling AN, Lee MJ. A survey of interventional radiology awareness among final-year medical students in a European country. Cardiovasc Intervent Radiol. 2009;32(4):623-629.

18. Anzilotti K, Kamin DS, Sunshine JH, Forman HP. Relative attractiveness of diagnostic radiology: assessment with data from the National Residency Matching Program and comparison with strength of the job market. Radiology. 2001;221(1):87-91.

19. Arleo EK, Bluth E, Francavilla M, Straus CM, Reddy S, Recht M. Surveying fourth-year medical students regarding the choice of diagnostic radiology as a specialty. J Am Coll Radiol. 2016;13(2):188-195.

20. Shah S. Are curriculum changes the ideal method for increasing undergraduate exposure to tomorrow's specialties? Adv Med Educ Pract. 2015;6:153-154.

21. Royal College of Psychiatrists. Recruitment Strategy 2011-2016. Royal College of Psychiatrists; 2012. Available from: http://www.rcpsych. ac.uk/pdf/Recruitment\%20Strategy\%20-\%2010092013.pdf. Accessed November 16, 2017.

22. Teaching undergraduates radiology: a guidance paper for teachers of undergraduates. European Society of Radiology. 2015. Available from: https://www.myesr.org/sites/default/files/ESR_2015_Teaching_Undergraduates_web.pdf. Accessed September 15, 2017. 
23. UK undergraduate curriculum for interventional radiology. British Society of Interventional Radiology. 2014.

24. Pantoja E, Nagy F, Zambernard J. Clinical radiographs of the cadaver as a teaching aid in anatomy. Radiology. 1985;155(1):28.

25. Salajegheh A, Jahangiri A, Dolan-Evans E, Pakneshan S. A combination of traditional learning and e-learning can be more effective on radiological interpretation skills in medical students: a pre- and post-intervention study. BMC Med Educ. 2016;16:46.
26. Codd AM, Choudhury B. Virtual reality anatomy: is it comparable with traditional methods in the teaching of human forearm musculoskeletal anatomy? Anat Sci Educ. 2011;4(3):119-125.

27. Gould D. Using simulation for interventional radiology training. $\mathrm{Br} \mathrm{J}$ Radiol. 2010;83(991):546-553.

Advances in Medical Education and Practice

\section{Publish your work in this journal}

Advances in Medical Education and Practice is an international, peerreviewed, open access journal that aims to present and publish research on Medical Education covering medical, dental, nursing and allied health care professional education. The journal covers undergraduate education, postgraduate training and continuing medical education including emerging trends and innovative models linking education, research, and health care services. The manuscript management system is completely online and includes a very quick and fair peer-review system. Visit http://www.dovepress.com/testimonials.php to read real quotes from published authors.

Submit your manuscript here: http://www.dovepress.com/advances-in-medical-education-and-practice-journal 